\title{
Association between long non-coding RNA and human rare diseases (Review)
}

\author{
JIN-HUA HE, ZE-PING HAN and YU-GUANG LI \\ Department of Laboratory, Central Hospital of Panyu District, Guangzhou, Guangdong 511400, P.R. China
}

Received July 30, 2013; Accepted September 23, 2013

DOI: $10.3892 /$ br.2013.191

\begin{abstract}
Long non-coding RNAs (lncRNAs) are untranslated transcripts with longer than 200 nucleotides (nt), which possess many of the structural characteristics of mRNAs, including a poly A tail, 5'-capping, and a promoter structure, but no conserved open reading frame. Moreover, lncRNA expression patterns change during differentiation and exhibit a variety of splicing patterns. Many lncRNAs are expressed at specific times and in specific tissues during development. It has been proposed that lncRNAs are involved in the epigenetic regulation of coding genes, and thus exert a powerful effect on a number of physiological and pathological processes, including the pathogenesis of many human rare diseases.
\end{abstract}

\section{Contents}

1. Introduction

2. The association between long non-coding RNA and human rare diseases

3. Prader-Willi syndrome

4. Angelman syndrome

5. Beckwith-Wiedemann syndrome

6. DiGeorge syndrome

7. Down's syndrome

8. Klinefelter's syndrome

9. Restless legs syndrome

10. Silver-Russell syndrome

11. West syndrome

12. Fragile X syndrome

13. Cat eye syndrome

14. Blepharophimosis syndrome

15. Conclusions

Correspondence to: Dr Yu-Guang Li, Department of Laboratory, Central Hospital of Panyu District, 8 Fuyu Dong Road, Guangzhou, Guangdong 511400, P.R. China

E-mail: lyg_py@126.com

Key words: long non-coding RNA, human rare diseases, association

\section{Introduction}

At present, five classes of long non-coding RNAs (lncRNAs) are known: anti-sense lncRNAs, intronic non-coding RNAs, large intergenic non-coding RNAs (lincRNAs), promoter-associated IncRNAs, and untranslated region (UTR)-associated lncRNAs (1). These lncRNAs are involved in a variety of vital regulation processes, including X-chromosome inactivation, genomic imprinting, chromatin modification, transcriptional activation, transcriptional interference, and nuclear transport, suggesting a possible involvement in diseases (2). lncRNAs are aberrantly expressed in several complex disorders and appear to be directly linked to the incidence of some diseases (3). This review summarizes the potential association between lncRNAs and human rare diseases.

\section{The association between long non-coding RNA and} human rare diseases

In differentiation and development processes, the dysfunction of non-coding RNA often leads to disease. Abnormality of lncRNA might affect DNA methylation, histone modification, and chromatin remodeling in various ways. Moreover, as a precursor of microRNA, lncRNAs play an important role in the initiation and progression of disease. Recent studies $(4,5,6,7)$ have found that $\mathrm{C} 15$ orf2, H19, Ube3a-as, and DGCR5 are associated with rare diseases (Table I).

\section{Prader-Willi syndrome}

Prader-Willi syndrome (PWS) is a neurogenetic disorder that results from loss of the paternal contribution of a $1.5-\mathrm{Mb}$ imprinted region on the proximal long arm of chromosome 15. PWS is characterized by neonatal muscular hypotonia and failure to thrive, hyperphagia and obesity starting in early childhood, as well as hypogonadism, short stature, small hands and feet, sleep apnea, behavioral problems and mild to moderate mental retardation (8). C15orf2 is a testis-specific gene that maps between $N D N$ and $S N U R F-S N R P N$ and is expressed by the two alleles. The novel genes Prader-Willi region non-protein-coding RNA 1 and 2 are located between NDN and C15orf2. PWRN2 is expressed only in testis and is biallelic (4). PWRN1 is biallelically expressed in testis and kidney. Investigation of $C 15$ orf 2 revealed that this gene is also expressed in the fetal brain but is monoallelic. Therefore, 
Table I. Long non-coding RNAs and human rare diseases.

\begin{tabular}{lcccc}
\hline Name & Location & Length $(\mathrm{bp})$ & Dysfunction type & Associated diseases \\
\hline C15orf2 & Chr15: 24920541-24928593 & 8053 & Expression & Prader-Willi syndrome \\
H19 & Chr11: 2016406-2019065 & 2559 & Epigenetics & Beckwith-Wiedemann \\
Ube3a-as & Unknown & Unknown & Locus & Angelman syndrome \\
KCNQ1OT1 & Chr11: 2661768-2721228 & 91671 & Epigenetics & Beckwith-Wiedemann syndrome \\
DGCR5 & Chr12: 18958027-18982141 & 3334 & Expression and mutation & DiGeorge syndrome \\
NRON & Chr9q33.3: 129270966-129481601 & 2730 & Expression & Down's syndrome \\
XIST & ChrX: $73040495-73072588$ & 31093 & Epigenetics & Klinefelter's syndrome \\
Ak042766 & Unknown & 1029 & Expression & Restless legs syndrome \\
BX118339 & Chr6: 21486545-21512123 & 25578 & Mutation & West syndrome \\
ASFMR1 & ChrX: 146990949-147003676 & 3026 & Expression & Fragile X syndrome \\
CECR3 & Chr22: $17737750-17747623$ & 1915 & Expression & Cat eye syndrome \\
CECR9 & Chr22: $17809924-17810122$ & 198 & Expression & Cat eye syndrome \\
BPESC1 & Chr3: 138823027-138844009 & 3518 & Mutation & Blepharophimosis syndrome \\
\hline
\end{tabular}

PWRN1 and C15orf2 may be involved in PWS. ZNF127 and ZNF127AS are imprinted genes that may be associated with some of the clinical features of the polygenic Prader-Willi syndrome (9).

\section{Angelman syndrome}

Angelman syndrome is a complex genetic disorder primarily affecting the nervous system. Characteristic features of this condition include delayed development, intellectual disability, severe speech impairment, and problems with movement and balance (5). Ube3a-as is a IncRNA transcribed antisense to the maternally expressed $U b e 3 a$ gene, a candidate gene for Angelman syndrome, suggesting that Ube $3 a-a s$ may be responsible for the repression of paternal Ube3a expression (10).

\section{Beckwith-Wiedemann syndrome}

Beckwith-Wiedemann syndrome (BWS) is an imprinting disorder characterized by overgrowth, tumor predisposition, and congenital malformations. Approximately $85 \%$ of reported BWS cases are sporadic, while the remaining $15 \%$ are familial. BWS is caused by epigenetic or genomic alterations that disrupt genes in one or both of the two imprinted domains on chromosome 11p15.5 (11). Genetic alterations of H19 and LITI distinguish patients with BWS from those with cancer and birth defects. Microdeletions in the human $H 19$ DMR result in loss of IGF2 imprinting and BWS (6). The LIT1 CpG island acts as a negative regulator in cis for coordinate imprinting at the centromeric domain, suggesting a role for the LITI locus in a BWS pathway, which results in the functional inactivation of $p 57$ (KIP2) (12). In vitro fertilization may increase the risk of BWS, and it may be linked to the abnormal imprinting of the KCNIOT gene, suggesting that LITI (KCNQIOT1) plays an important role in the development of BWS (13). In BWS, 50\% of patients show loss of DNA methylation accompanied by loss of histone $\mathrm{H} 3$ Lys9 dimethylation on maternal KCNQ1OT-DMR, known as imprinting disruption, which causes the decreased expression of $C D K N 1 C$ (14). Imprinting disruption of the CDKN1C/ KCNQ1OT1 domain is involved in the development of BWS and cancer, and it changes the maternal epigenotype to the paternal type, resulting in a decrease of CDKNIC expression (15).

\section{DiGeorge syndrome}

The microdeletion $22 \mathrm{q} 11.2$ syndrome is one of the more common human deletion syndromes (1 in 4,000 live births) and encompasses several clinical entities. Approximately $35-90 \%$ of patients clinically diagnosed with DiGeorge syndrome, including cardiac abnormalities such as interrupted aortic arch, truncus arteriosus and tetralogy of Fallot, and $80-100 \%$ of those with velocardiofacial syndrome, including pharyngeal dysfunction, cardiac anomalies and dysmorphic facies, have this hemizygous deletion (16). The essential transcriptional repressor element 1-silencing transcription factor (REST) is crucial in the development of human disease by regulating a large cohort of neural genes (17). The DiGeorge syndrome-associated non-coding RNA, $D G C R 5$, is repressed by REST through a proximal upstream binding site, and disrupted by the DiGeorge syndrome patient breakpoint (7).

\section{Down's syndrome}

Trisomy 21 or Down syndrome (DS), is the most frequent and recognizable cause of intellectual disabilities. DS is a major cause of mental retardation and congenital heart disease. Besides a characteristic set of facial and physical features, DS is associated with congenital anomalies of the gastrointestinal tract, an increased risk of leukemia, immune system defects and an Alzheimer-like dementia. Moreover, DS is a model for 
the study of human aneuploidy (18). NRON (Homo sapiens non-protein coding RNA, repressor of NFAT non-coding RNA) is a lncRNA that mediates the cytoplasmic to nuclear shuttling of the NFAT transcription factor. In animal models, deregulation of the DSCRI and DYRK1A genes acts synergistically to prevent nuclear occupancy of NFATc transcription factors, leading to reduced $N F A T c$ activity and to a number of features of DS, suggesting a potential link between NRON activity and DS pathophysiology (19).

\section{Klinefelter's syndrome}

Klinefelter's syndrome, also known as 47,XXY or XXY syndrome, is a genetic disorder in which there is at least one extra $\mathrm{X}$ chromosome to a standard human male karyotype, with a total of 47 chromosomes rather than the 46 found in genetically normal humans. The presence of an isochromosome $\mathrm{Xq}$ in Klinefelter syndrome (KS) is an apparently rare condition (20). In all cases reported thus far, patients showed the classical phenotype. Severe XIST hypomethylation clearly distinguishes (SRY+) 46,XX-maleness from Klinefelter syndrome (21). Quantitative RT-PCR demonstrated that an active XIST RNA expression in blood lymphocytes of Klinefelter patients, comparable to that observed in the control females although $>30,000$-fold higher than the control males. The higher expression of XIST provides a clue to the diagnosis of Klinefelter syndrome (22).

\section{Restless legs syndrome}

Approximately $65 \%$ of restless legs syndrome (RLS) patients, particularly those with an early onset of symptoms, have at least one first-degree relative with the disease. The concordance rate between monozygotic twins has also been reported to be high. Most pedigrees suggest an autosomal dominant inheritance, although recessive models have been proposed as well. Sequence variants have also been proposed, in or around genes on $6 \mathrm{p}, 2 \mathrm{p}$, or $15 \mathrm{q}$ (23). The most significant gene identified currently is Meis1. A recent study (24) suggested that the predisposition to RLS results from a reduced expression of Meis 1 mediated by intronic cis-regulatory elements. Of note, in the developing mouse brain, Meisl is co-expressed in the developing cerebellar granule cell layer along with a genomically-associated lncRNA AK042766 (24).

\section{Silver-Russell syndrome}

Silver-Russell syndrome (SRS) is a genetically and clinically heterogeneous disease that is mainly characterized by preand postnatal growth restriction. The typical SRS phenotype includes a relative macrocephaly, a triangular shaped face, body asymmetry, clinodactyly of the fifth finger and other less constant features (25), as well as epigenetic mutations of the imprinted IGF2-H19 domain in SRS. The majority of the patients with methylation abnormalities showed hypomethylation at both the $H 19$ and IGF2 genes. However, it was also identified that SRS patients with hypomethylation were restricted to the $H 19$ or $I G F 2$ gene. Epimutations were also identified in siblings of normal parents, most likely reflecting germ cell mosaicism in the fathers. In one family, epimutation was identified in an affected father and his likewise affected daughter (26).

\section{West syndrome}

West syndrome is a disabling, age-related epileptic encephalopathy that may be attributed to different aetiologies. This syndrome is characterized by a unique seizure-type of development (27). A de novo balanced $\mathrm{t}(2 ; 6)(\mathrm{p} 15 ; \mathrm{p} 22.3)$ in a patient with West syndrome disrupts a lncRNA, and BX118339 spans the breakpoint of chromosome 6 . It could be hypothesized that disruption of this non-coding transcript plays a role in the pathogenesis of the patient (28).

\section{Fragile $X$ syndrome}

Fragile $\mathrm{X}$ syndrome, an X-linked dominant disorder with reduced penetrance, is associated with intellectual and emotional disabilities ranging from learning problems to mental retardation, and from mood instability to autism (29). Homo sapiens FMRl antisense RNA 1 (FMRl-ASl), transcript variant 1 , is a non-coding RNA. ASFMRl is silenced in FXS patients and upregulated in pre-mutation carriers, suggesting that a common process is responsible for regulating the expression of these transcripts (30). A primate-specific non-coding RNA transcript $(2.4 \mathrm{~kb})$ that resides upstream likely shares a bidirectional promoter with FMR1. FMR4 is a product of RNA polymerase II and has a similar half-life to FMR1. FMR4 is also silenced in FXS patients because of a CGG expansion repeat in the 5' UTR of the FMR1 gene but is upregulated in pre-mutation carriers (31).

\section{Cat eye syndrome}

Cat eye syndrome (CES), or Schmid-Fraccaro syndrome, is a rare condition caused by the short arm (p) and a small section of the long arm (q) of human chromosome 22 in trisomic or tetrasomic forms instead of the normal disomic form. CES is actually a genomic disorder (32), with a homo sapiens CES chromosome region, a candidate 3 (non-protein coding) (CECR3) for non-coding RNA. It was found to be a $1.1-\mathrm{Mb}$ region of human chromosome $22 \mathrm{q}$ containing the dosage-sensitive gene(s) responsible for CES and the $450-\mathrm{kb}$ homologous region on mouse chromosome 6 (33).

\section{Blepharophimosis syndrome}

The blepharophimosis syndrome (BPES) is a rare genetic disorder characterized by blepharophimosis, ptosis, epicanthus inversus and telecanthus (34). The BPES syndrome has been mapped to $3 \mathrm{q} 23$. Homo sapiens blepharophimosis, epicanthus inversus and ptosis, candidate 1 (non-protein coding) non-coding RNA (BPESC1), BPESC1, is disrupted by a balanced chromosomal translocation, $\mathrm{t}(3 ; 4)(\mathrm{q} 23 ; \mathrm{p} 15.2)$, in a patient with BPES. It plays a significant role in the pathogenesis of BPES (35). BPES is driven by dysregulation of the FOXL2 gene, and a number of extragenic mutations have been reported in patients. A particular deletion occurring $283 \mathrm{~kb}$ away from FOXL2 disrupts a lncRNA, PISRT1 (Homo sapiens polled intersex syndrome-regulated transcript 1 ), which was 
shown by chromatin confirmation capture to be a physical loop with FOXL2 (36).

\section{Conclusions}

A total of 195 functional lncRNAs have been identified (http://ncrnadb.com, accessed April 2, 2013). However, the number of currently identified functional lncRNAs is a small fraction of that estimated by bioinformatics. Given that lncRNAs are capable of regulating the expression of coding genes, aberrant lncRNA expression is likely to cause disorders associated with dysregulation of the specific proteins. Thus, studies of lncRNA regulation and its function may provide new insights into the disease etiology, while diseases associated with known protein pathways may reveal the function of lncRNA. Our study suggests that lncRNAs are involved in the progression of this type of disease, through the up- or downregulation of specific mRNAs, methylation and regulation of specific gene polymorphisms.

Despite the present advances, the study of lncRNA is still in its infancy and the association between lncRNAs and the majority of other diseases (if any) remains to be determined. To explore lncRNAs as markers for clinical diagnosis, studies on IncRNA should compare the IncRNA expression profiles between healthy subjects and patients with the genetic disorders. Gene knockout, RNA interference, and transgenic strategies are likely to reveal more information regarding lncRNA regulation and function, leading to novel treatments for the diseases. Consequently, the study of lncRNA may be of great significance for understanding of the intricate and multi-level regulatory systems in the development, for prevention and treatment of human genetic diseases, and for identification of the principles in evolution.

\section{References}

1. Caley DP, Pink RC, Trujillano D and Carter DR: Long noncoding RNAs, chromatin, and development. Sci World J 8: 90-102, 2010.

2. Mercer TR, Dinger ME and Mattick JS: Long non-coding RNAs: insights into functions. Nat Rev Genet 10: 155-159, 2009.

3. Øromua, Derrien T, Beringer M, et al: Long noncoding RNAs with enhancer-like function in human cells. Cell 143: 46-58. 2010.

4. Buiting K, Nazlican H, Galetzka D, Wawrzik M, Gross S and Horsthemke B: C15orf2 and a novel noncoding transcript from the Prader-Willi/Angelman syndrome region show monoallelic expression in fetal brain. Genomics 89: 588-595, 2007.

5. Bodo C: Neurodevelopmental disorders: mechanistic insights into Angelman's syndrome. Nat Rev Neurosci 11: 298, 2010.

6. Reik W, Brown KW, Slatter RE, Sartori P, Elliott M and Maher ER: Allelic methylation of H19 and IGF2 in the Beckwith-Wiedemann syndrome. Hum Mol Genet 3: 1297-1301, 1994.

7. Johnson R, Teh CH, Jia H, Vanisri RR, Pandey T, Lu ZH, Buckley NJ, Stanton LW and Lipovich L: Regulation of neural macroRNAs by the transcriptional repressor REST. RNA 15: 85-96, 2009.

8. Qureshi IA, Mattick JS and Mehler MF: Long non-coding RNAs in nervous system function and disease. Brain Res 1338: 20-35, 2010.

9. Jong MT, Gray TA, Ji Y, Glenn CC, Saitoh S, Driscoll DJ and Nicholls RD: A novel imprinted gene, encoding a RING zinc-finger protein, and overlapping antisense transcript in the Prader-Willi syndrome critical region. Hum Mol Genet 8: 783-793, 1999.

10. Greer PL, Hanayama R, Bloodgood BL, et al: The Angelman Syndrome protein Ube3A regulates synapse development by ubiquitinating arc. Cell 140, 704-716, 2010.
11. Choufani S, Shuman C and Weksberg R: Beckwith-Wiedemann syndrome. Am J Med Genet C Semin Med Genet 154: 343-354, 2010.

12. Sparago A, Cerrato F, Vernucci M, Ferrero GB, Silengo MC and Riccio A: Microdeletions in the human H19 DMR result in loss of IGF2 imprinting and Beckwith-Wiedemann syndrome. Nat Genet 36: 958-960, 2004.

13. Arima T, Kamikihara $T$, Hayashida $T$, Kato $K$, Inoue $T$, Shirayoshi Y,Oshimura M, SoejimaH, Mukai T and WakeN: ZAC, LIT1 (KCNQ1OT1) and p57KIP2 (CDKN1C) are in an imprinted gene network that may play a role in Beckwith-Wiedemann syndrome. Nucleic Acids Res 33: 2650-2660, 2005.

14. Horike S, Mitsuya K, Meguro M, Kotobuki N, Kashiwagi A, Notsu T, Schulz TC, Shirayoshi Y and Oshimura M: Targeted disruption of the human LIT1 locus defines a putative imprinting control element playing an essential role in Beckwith-Wiedemann syndrome. Hum Mol Genet 9: 2075-2083, 2000.

15. Higashimoto K, Soejima H, Saito T, Okumura K and Mukai T: Imprinting disruption of the CDKN1C/KCNQ1OT1 domain: the molecular mechanisms causing Beckwith-Wiedemann syndrome and cancer. Cytogenet Genome Res 113: 306-312, 2006.

16. González W and Bautista RE: Seizures and EEG findings in an adult patient with DiGeorge syndrome: a case report and review of the literature. Seizure 18: 648-651, 2009.

17. Sutherland HF, Wadey R, McKie JM, Taylor C, Atif U, Johnstone KA, Halford S, Kim UJ, Goodship J, Baldini A and Scambler PJ: Identification of a novel transcript disrupted by a balanced translocation associated with DiGeorge syndrome. Am J Hum Genet 59: 23-31, 1996.

18. Mégarbané A, Noguier F, Stora S, Manchon L, Mircher C, Bruno R, Dorison N, Pierrat F, Rethoré MO, Trentin B, Ravel A, Morent M, Lefranc G and Piquemal D: The intellectual disability of trisomy 21: differences in gene expression in a case series of patients with lower and higher IQ. Eur J Hum Genet: Feb 20, 2013 (Epub ahead of print).

19. Willingham AT, Orth AP, Batalov S, Peters EC, Wen BG, Aza-Blanc P, Hogenesch JB and Schultz PG: A strategy for probing the function of noncoding RNAs finds a repressor of NFAT. Science 309: 1570-1573, 2005.

20. Poplinski A, Wieacker P, Kliesch S and Gromoll J: Severe XIST hypomethylation clearly distinguishes (SRY+) 46,XX-maleness from Klinefelter syndrome. Eur J Endocrinol 162: 169-175, 2010.

21. Visootsak J and Graham JM Jr: Klinefelter syndrome and other sex chromosomal aneuploidies. Orphanet J Rare Dis 1: $42,2006$.

22. Stabile M, Angelino T, Caiazzo F, et al: Fertility in a $\mathrm{i}(\mathrm{Xq})$ Klinefelter patient: importance of XIST expression level determined by qRT-PCR in ruling out Klinefelter cryptic mosaicism as cause of oligozoospermia. Mol Hum Reprod 14: 635-640, 2008

23. Ovallath S and Deepa P: Restless legs syndrome. J Parkinsonism Restless Legs Syndr 2: 49-57, 2012.

24. Ponjavic J, Oliver PL, Lunter G and Ponting CP: Genomic and transcriptional co-localization of protein-coding and long non-coding RNA pairs in the developing brain. PLoS Genet 5: e1000617, 2009.

25. Eggermann T, Begemann M, Spengler S, Schröder C, Kordass U and Binder G: Genetic and epigenetic findings in Silver-Russell syndrome. Pediatr Endocrinol Rev 8: 86-93, 2010.

26. Bartholdi D, Krajewska-Walasek M, Õunap K, et al: Epigenetic mutations of the imprinted IGF2-H19 domain in Silver-Russell syndrome (SRS): results from a large cohort of patients with SRS and SRS-like phenotypes. J Med Genet 46: 192-197, 2009.

27. Japaridze N, Muthuraman M, Moeller F, Boor R, Anwar AR, Deuschl G, Stephani U, Raethjen J and Siniatchkin M: Neuronal networks in west syndrome as revealed by source analysis and renormalized partial directed coherence. Brain Topogr 6: 157-170, 2013.

28. Vandeweyer G, Van der Aa N, Ceulemans B, van Bon BW, Rooms L and Kooy RF: A de novo balanced $\mathrm{t}(2 ; 6)(\mathrm{p} 15 ; \mathrm{p} 22.3)$ in a patient with West Syndrome disrupts a lnc-RNA. Epilepsy Res 99: 346-349, 2012.

29. Garber KB, Visootsak J and Warren ST: Fragile X syndrome. Eur J Hum Genet 16: 666-672, 2008.

30. Ladd PD, Smith LE, Rabaia NA, Moore JM, Georges SA, Hansen RS, Hagerman RJ, Tassone F, Tapscott SJ and Filippova GN: An antisense transcript spanning the CGG repeat region of FMR1 is upregulated in premutation carriers but silenced in full mutation individuals. Hum Mol Genet 16: 3174-3187, 2007. 
31. Khalil AM, Faghihi MA, Modarresi F, Brothers SP and Wahlestedt C: A novel RNA transcript with antiapoptotic function is silenced in fragile X syndrome. PLoS One 3: e1486, 2008.

32. Rosias PR, Sijstermans JM, Theunissen PM, et al: Phenotypic variability of the cat eye syndrome. Case report and review of the literature. Genet Couns 12: 273-282, 2001.

33. Footz TK, Brinkman-Mills P, Banting GS, Maier SA, Riazi MA, Bridgland L, Hu S, Birren B, Minoshima S, Shimizu N, Pan H, Nguyen T, Fang F, Fu Y, Ray L, Wu H, Shaull S, Phan S, Yao Z, Chen F, Huan A, Hu P, Wang Q, Loh P, Qi S, Roe BA and McDermid HE: Analysis of the cat eye syndrome critical region in humans and the region of conserved synteny in mice: a search for candidate genes at or near the human chromosome 22 pericentromere. Genome Res 11: 1053-1070, 2001.
34. Alao MJ, Lalèyè A, Lalya F, Hans Ch, Abramovicz M, Morice-Picard F, Arveiler B, Lacombe D and Rooryck C: Blepharophimosis, ptosis, epicanthus inversus syndrome with translocation and deletion at chromosome $3 \mathrm{q} 23$ in a black African female. Eur J Med Genet 55: 630-634, 2012.

35. De Baere E, Fukushima Y, Small K, Udar N, Van Camp G, Verhoeven K, Palotie A, De Paepe A and Messiaen L: Identification of BPESC1, a novel gene disrupted by a balanced chromosomal translocation, $\mathrm{t}(3 ; 4)(\mathrm{q} 23 ; \mathrm{p} 15.2)$, in a patient with BPES. Genomics 68: 296-304, 2000.

36. Hung $\mathrm{T}$ and Chang HY: Long noncoding RNA in genome regulation: prospects and mechanisms. RNA Biol 7: 582-585, 2010. 